\title{
FACTORES PARA LA DECISIÓN DEL PARTO VAGINAL EN MUJERES CON CESÁREA PREVIA EN LA CLÍNICA PARTICULAR CORI GYN, DISTRITO LOS OLIVOS 2015
}

\author{
DECISION FACTORS TO THE VAGINAL DELIVERIES IN WOMEN WITH CESAREA \\ PREVIA AT THE PARTICULAR CORI GYN CLINIC THE OLIVES DISTRICT 2015
}

\section{María Caldas Herrera ${ }^{1}$ - Cynthia Carmen Carrión Barreto²}

1 Obstetriz.- Docente de la Escuela profesional de Obstetricia de la Universidad Alas Peruanas

2 Tesista de la Escuela profesional de Obstetricia de la Universidad Alas Peruanas

Email: S.decanatouap@gmail.com

TL. +51987220183

\section{RESÚMEN}

Objetivo determinar los factores para la decisión del parto vaginal en mujeres con cesárea previa atendidas en la Clínica particular CORI GYN, distrito Los Olivos 2015. Material y método: Estudio descriptivo retrospectivo de corte transversal. Se estudió a 90 mujeres con cesárea previa. Para el análisis se utilizó la medida central, dispersión, así como frecuencias absolutas y relativas. Resultados:la edad promedio de las mujeres con cesárea previa fue 29,6 años, donde la edad $\leq 35$ años fue el predominante $(96,7 \%)$, asimismo el grado de instrucción fue mayormente universitario $(54,4 \%)$ y el estado civil casada $(70,0 \%)$. Entre los factores maternos más frecuentes asociados a la decisión del parto vaginal fue el período intergenésico de 2 - 5 años (60,0\%), parto vaginal previo en un 53,3\%, ser multigesta (51,1\%) e inicio de parto espontáneo (92,2\%). En el 93,3\% de los casos la decisión de la vía de culminación fue tomada por el médico. Respecto a las complicaciones, el 6,7\% presentaron complicaciones en el puerperio inmediato (retención de restos placentarios, sangrado e infección de episiotomía) y el 20,0\% presentaron complicaciones en el puerperio mediato (fiebre, infección de episiotomía y alza térmica). En los factores fetales asociados a la decisión del parto vaginal se encontró que la edad gestacional fue de 38 a 40 semanas con un peso promedio de 3,296 gramos. Las conclusiones a las que se le llegó fue que los 
factores más frecuentes asociados a la decisión de parto por vía vaginal en mujeres con cesárea previa fueron el período intergenésico entre 2 y 5 años, parto vaginal previo, ser multigesta, además de la edad gestacional a término y ponderado del peso fetal normal.

Palabras clave: parto vaginal, cesárea previa.

\section{SUMMARY}

This study was planned with the objective to determine the factors to the decision of vaginal birth in women with previous cesarean treated in private clinic CORI GYN, Los Olivos 2015. Material and Methods: Observational, descriptive, retrospective, cross-sectional study. We studied 90 women with previous cesarean. For the analysis the central measure, dispersion was used; as well as absolute and relative frequencies. In the study results it shows that the average age of women with previous cesarean was 29.6 years, where age $\leq 35$ years was predominant $(96.7 \%)$, also the level of education was mostly college (54.4\%) and married marital status (70.0\%). Among the most common maternal factors associated with the decision of vaginal delivery were intergenesic period of 2 to 5 years $(60.0 \%)$, previous vaginal delivery in $53.3 \%$, being multigesta (51.1\%) and starting spontaneous delivery (92.2\%). In 93.3\% of cases the decision on the route of culmination was taken by the doctor. Regarding complications, $6.7 \%$ had complications in the immediate postpartum period (retention of placental remains, bleeding and infection of episiotomy) and $20.0 \%$ had complications taken during mediate puerperium (fever, infection episiotomy and thermal rise). In fetal factors associated with vaginal delivery decision it found that gestational age was 38 to 40 weeks with an average weight of 3,2963 grams. The conclusions reached were that the most common factors associated with the decision of vaginal delivery in women with previous cesarean were intergenesic period between 2 and 5 years prior vaginal delivery, be multigesta, onset of spontaneous labor besides gestational age; and weighted normal the fetus.

Key word: vaginal birth, previous cesarean

\section{INTRODUCCIÓN}

En la actualidad, el parto mediante cesárea es cada vez más común. La mayoría de las veces el parto vaginal es posible en una mujer con antecedente de cesárea, a pesar que existe un mayor riesgo de rotura uterina; sin embargo se considera seguro tanto para la madre como para el feto. En contraparte, la cesárea electiva rutinaria para el segundo parto de una mujer con una cesárea previa genera un exceso de morbilidad y mortalidad materna además de un alto costo para el sistema sanitario. La operación cesárea expone a mayor peligro de morbimortalidad 
materno fetal que el parto vaginal no complicado, además de los riesgos propios de la anestesia. En gestantes de alto riesgo además del estado de alarma fisiológico por la cesárea hay aumento de morbimortalidad de la madre y del niño en el período de recuperación, por complicaciones infecciosas, hemorrágicas y pulmonares. ${ }^{1}$ Actualmente existe una fuerte tendencia al alza en las tasas de cesárea, en nuestro país se observa una cifra superior a lo recomendado por la Organización Mundial de la Salud, a pesar que esta misma institución asevera que no existen fundamentos que justifiquen una taza superior al 15\%, describiéndose como mala calidad de atención obstétrica cifras superiores. Considerando esta problemática, la presente investigación tiene el objetivo de determinar los factores para la decisión del parto vaginal en mujeres con cesárea, cuyos resultados permitirán brindar una atención más humanizada que garantice una atención de calidad para el binomio madre niño

\section{MATERIAL Y MÉTODOS}

El presente estudio es descriptivo, retrospectivo y de corte transversal. De acuerdo a la intervención del investigador, el estudio es descriptivo, porque se realizó un análisis univariado de los factores para la decisión del parto vaginal en mujeres con antecedente de cesárea previa. Según las veces en que se mide las variables el estudio es transversal porque la recopilación de los datos se realizó en una sola ocasión durante el mismo período de estudio planteado. De acuerdo a la planificación de recopilación de datos, el estudio es retrospectivo porque el evento de interés ya ocurrió por lo que la información fue tomada de fuentes secundarias. ${ }^{40}$

El método es aplicativo, porque nos permite solucionar problemas, en el presente estudio se describe los factores para la decisión del parto vaginal en pacientes con cesárea previa con la finalidad de detectar cuál de ellos es más frecuente en nuestra realidad hospitalaria y así intervenir de manera efectiva.

El método es cuantitativo, porque se vale de números para analizar la información con un determinado nivel de error y nivel de confianza.

El universo estuvo conformada por 241 mujeres que tuvieron parto vaginal en el año 2015. La muestra fue censal es decir todas las mujeres que fueron sometidas a parto vaginal con cesárea previa.

Criterios de Inclusión • Historia clínica de todas las mujeres con parto por vía vaginal y antecedente de cesárea previa (una vez). - Historia clínica de las mujeres atendidas en la Clínica particular Cori Gyn entre enero a diciembre de 2015 . Historia clínica en buen estado (legible, completo y accesible). Criterios de Exclusión - Historia clínica de mujeres con antecedente de dos o más intervenciones quirúrgicas por cesárea. • Historia clínica de mujeres con edad gestacional menor a 37 semanas. • Historia clínica de mujeres con embarazo múltiple. 
Para el levantamiento de la información se elaboró una ficha de recolección de datos, la misma que fue sometida a juicio de tres expertos quienes dieron la conformidad con la firma respectiva en la hoja de evaluación. La ficha de recolección se caracteriza por tres partes que permiten dar respuesta a los objetivos de la investigación. Además se distribuyen de la siguiente manera: 16 items de datos generales: Factores maternos: lo componen 8 ítems Factores fetales: lo componen 7 ítems El instrumento ha sido elaborado por el investigador en base a la revisión de la literatura sobre el tema y se incluyen variables identificadas con mayor frecuencia en los antecedentes.

Los datos recopilados en las fichas de registro fueron procesados en el programa estadístico SPSS versión 20, donde se codificaron y recategorizaron las variables según el cuadro de variables y se presentaron en cuadros estadísticos y gráficos según los resultados obtenidos.

\section{RESULTADOS}

Luego de haber procesado los datos se encontraron los siguientes resultados:

\section{Tabla 1.}

\section{DATOS GENERALES EN PACIENTES CON ANTECEDENTES DE CESÁREA PREVIA}

\begin{tabular}{lcc}
\hline Datos generales & \pm SD & Min- Max \\
\hline Edad (años) & $29,6 \pm 3,2$ & $22-39$ \\
& N & $\%$ \\
\hline 35 años & 87 & $96,7 \%$ \\
$>35$ años & 3 & $3,3 \%$ \\
Grado de Instrucción & & \\
Primaria & 1 & $1,1 \%$ \\
Secundaria & 25 & $27,8 \%$ \\
Técnico & 15 & $16,7 \%$ \\
Universitario & 49 & $54,4 \%$ \\
Estado Civil & & \\
Soltera & 1 & $1,1 \%$ \\
Casada & 63 & $70,0 \%$ \\
Conviviente & 26 & $28,9 \%$ \\
Ocupación & & \\
Profesional de Salud & 30 & $33,3 \%$ \\
Ama de casa & 19 & $21,1 \%$ \\
Administración- Contabilidad & 10 & $11,1 \%$ \\
Psicología & 6 & $6,7 \%$ \\
Docencia & 5 & $5,6 \%$ \\
Otros & 20 & $22,2 \%$ \\
Lugar de procedencia & & \\
Lima- Callao & 55 & $38,9 \%$ \\
Resto país & 35 & $100,0 \%$ \\
\hline Total & & \\
\hline
\end{tabular}

Fuente: Ficha de recolección de datos (historia clínica de gestantes con cesárea previa) en la Clínica particular CORI GYN en el año 2015. 
En la tabla $\mathrm{N}^{\circ} 1$ se observa que la edad promedio de las mujeres fue 29,6 años, donde la edad materna menor o igual a 35 años fue el predominante $(96,7 \%)$. El grado de instrucción universitario fue el más frecuente con (54,3\%) seguido de la secundaria $(28,7 \%)$. En -cuanto al estado civil las mujeres con cesárea previa mayormente fueron casadas $(70,0 \%)$, respecto al lugar de procedencia, se observa que el $61,1 \%$ procedía de Lima- Callao y el $38,9 \%$ del resto del país (Cajamarca, Chiclayo, Ica, entre otros departamentos del país).

TABLA 2. FACTORES MATERNOS: ANTECEDENTES GINECO-OBSTÉTRICOS EN PACIENTES CON ANTECEDENTES DE CESAREA PREVIA

\begin{tabular}{lcc}
\hline Antecedentes & N & $\%$ \\
\hline Período intergenésico & & \\
2 - 5 años & 54 & $60,0 \%$ \\
$>5$ años & 36 & $40,0 \%$ \\
Parto vaginal previo & & \\
Si & 48 & $53,3 \%$ \\
No & 42 & $46,7 \%$ \\
Antecedente de aborto & & \\
Si & 43 & $47,8 \%$ \\
No & 47 & $52,2 \%$ \\
$\quad$ Principales motivos de cesárea previa & & \\
Sufrimiento fetal agudo & 20 & $22,1 \%$ \\
Macrostomia fetal & 11 & $12,2 \%$ \\
Preeclampsia & 7 & $7,8 \%$ \\
Feto podálico & 7 & $7,8 \%$ \\
Otros & 55 & $50,1 \%$ \\
Total & 90 & $100,0 \%$ \\
\hline
\end{tabular}

Fuente: Ficha de recolección de datos (historia clínica de gestantes con cesárea previa) en la Clínica particular CORI GYN en el año 2015.

En la tabla $\mathrm{N}^{\circ} 2$, se observa que el período intergenésico entre 2 a 5 años corresponde en un $60,0 \%$. El $53,3 \%$ de las mujeres con cesárea previa tuvieron parto vaginal previo. Además el $47,8 \%$ presentaron antecedente de aborto. Entre los principales motivos que llevaron al parto vía abdominal previo fueron sufrimiento fetal $(22,1 \%)$ y la macrosomía fetal $12.2 \%$. 
Tabla 3. FACTORES MATERNOS: GINECO-OBSTÉTRICO ACTUAL EN PACIENTES CON ANTECEDENTES DE CESÁREA PREVIA.

\begin{tabular}{lcc}
\hline Características Gineco-obstétricas & \pm SD & Min- Max \\
\hline Controles Prenatales & $9,9 \pm 2,1$ & $6-14$ \\
Gestaciones & & \\
Segundigesta & 26 & $28,9 \%$ \\
Multigesta & 64 & $71,1 \%$ \\
Inicio de parto espontáneo & & \\
Si & 83 & $92,2 \%$ \\
No & 7 & $7,8 \%$ \\
Decisión vía de culminación & & \\
Médico & 84 & $93,3 \%$ \\
Gestante & 6 & $6,7 \%$ \\
Total & 90 & $100,0 \%$ \\
\hline
\end{tabular}

Fuente: Ficha de recolección de datos (historia clínica de gestantes con cesárea previa) en la Clínica particular CORI GYN en el año 2015.

En la tabla $\mathrm{N}^{\circ} 3$ : El control prenatal promedio fue $9,9 \pm 2,1$ controles, el $71,1 \%$ de las mujeres eran multigestas y el $28,9 \%$ eran segundigestas, el inicio de parto espontáneo, ocurrió en 9 de cada 10 mujeres, la decisión de la vía de culminación de parto fue mayormente del médico con $93.2 \%$

Tabla 4. CARACTERÍSTICAS DEL TRABAJO DE PARTO EN PACIENTES CON ANTECEDENTES DE CESÁREA PREVIA

\begin{tabular}{lrc}
\hline Características de trabajo de parto & \pm SD & Min- Max \\
\hline Tiempo de período de dilatación (horas) & $10,4 \pm 3,1$ & $5-19$ \\
Tiempo de período expulsivo (minutos) & $12,5 \pm 4,1$ & $2-24$ \\
Tiempo de período de alumbramiento (minutos) & $10,1 \pm 4,6$ & $5-38$
\end{tabular}

Fuente: Ficha de recolección de datos (historia clínica de gestantes con cesárea previa) en la Clínica particular CORI GYN en el año 2015.

En la tabla $N^{\circ} 4$ : se observa que el tiempo promedio del período de dilatación fue 10,4 horas, oscilando entre 5 y 19 horas; asimismo el tiempo promedio del período expulsivo fue 12,5 minutos y finalmente se encontró que el tiempo promedio del período de alumbramiento de las mujeres con antecedente de cesárea previa fue 10,1 minutos.

\section{Tabla 5. COMPLICACIONES EN EL PUERPERIO EN PACIENTES CON ANTECEDENTES DE} CESÁREA PREVIA 


\begin{tabular}{|c|c|c|}
\hline Complicaciones en el puerperio & $\pm \mathrm{SD}$ & Min- Max \\
\hline Tiempo de estancia post parto en la clínica (días) & $3,3 \pm 1,1$ & $2-6$ \\
\hline \multicolumn{3}{|l|}{ Complicaciones en el puerperio inmediato } \\
\hline $\mathrm{Si}$ & 6 & $6,7 \%$ \\
\hline No & 84 & $93,3 \%$ \\
\hline \multicolumn{3}{|l|}{ Complicaciones en el puerperio mediato } \\
\hline $\mathrm{Si}$ & 18 & $20,0 \%$ \\
\hline No & 72 & $80,0 \%$ \\
\hline Total & 188 & $100,0 \%$ \\
\hline
\end{tabular}

Fuente: Ficha de recolección de datos (historia clínica de gestantes con cesárea previa) en la Clínica particular CORI GYN en el año 2015.

Según la tabla $N^{\circ} 5$ se observa que el tiempo de estancia promedio fue 3,3 $\pm 1,1$ días, encontrándose entre 2 y 6 días. El $6,7 \%$ de las mujeres con antecedente de cesárea previa presentaron complicaciones en el puerperio inmediato entre ellas se tiene la retención de restos placentarios, sangrado, infección de episiotomía, entre otros; mientras que el $20,0 \%$ de las mujeres presentaron complicaciones en el puerperio mediato las cuales fueron la fiebre, infección episiotomía y alza térmica.

\section{Tabla 6. FACTORES FETALES EN PACIENTES CON ANTECEDENTES DE CESÁREA PREVIA}

\begin{tabular}{|c|c|c|}
\hline Factores fetales & \pm SD & Min- Max \\
\hline Edad gestacional (sem) & $39,2 \pm 0,6$ & $38-40$ \\
\hline Ponderado fetal (kg) & $3296,3 \pm 314,1$ & $2680-3980$ \\
\hline Distocia funicular & $\mathrm{N}$ & $\%$ \\
\hline $\mathrm{Si}$ & 20 & $22,2 \%$ \\
\hline No & 70 & $77,8 \%$ \\
\hline \multicolumn{3}{|l|}{ Malformaciones fetales } \\
\hline $\mathrm{Si}$ & 3 & $3,3 \%$ \\
\hline No & 87 & $96,7 \%$ \\
\hline Total & 90 & $100,0 \%$ \\
\hline
\end{tabular}

Fuente: Ficha de recolección de datos (historia clínica de gestantes con cesárea previa) en la Clínica particular CORI GYN en el año 2015.

En la Tabla $N^{\circ} 6$ se observa que la edad gestacional promedio de mujeres con cesárea previa fue $39,2 \pm 0,6$ semanas. Además el peso promedio del ponderado fetal fue $3296,3 \pm 314,1$ gramos. Por su parte, la distocia funicular se presentó en un $22,2 \%$ y las malformaciones fetales en las mujeres con cesárea previa fue del $3,3 \%$. 


\begin{tabular}{llc}
\hline Factores maternos y fetales & N & $\%$ \\
\hline Edad gestacional a término (38- 40) semanas & 90 & $100,0 \%$ \\
Ponderado fetal Normal & 90 & $100,0 \%$ \\
Decisión vía de culminación por el médico & 84 & $93,3 \%$ \\
Inicio de parto espontáneo & 83 & $92,2 \%$ \\
Multigesta & 64 & $71,1 \%$ \\
Período intergenésico (Entre 2 - 5 años) & 54 & $60,0 \%$ \\
Parto vaginal previo & 48 & $53,3 \%$ \\
\hline
\end{tabular}

Fuente: Ficha de recolección de datos (historia clínica de gestantes con cesárea previa) en la Clínica particular CORI GYN en el año 2015.

En la tabla 7 se observa los factores para la decisión del parto vaginal se encontró con mayor frecuencia a la edad gestacional a término $(100,0 \%)$, el ponderado fetal normal $(100,0 \%)$, la decisión vía culminación por el médico $(93,3 \%)$ y el inicio de parto espontaneo $(92,2 \%)$; mientras que ser multigesta $(71,1 \%)$, periodo intergenésico entre 2 y 5 años $(60,0)$ y parto de vaginal previo $(53,3 \%)$ fueron los menos frecuentes.

\section{DISCUSIÓN}

El manejo de las pacientes con cesárea previa anterior siempre ha sido tema de constantes debates, mientras en Europa, Asia y África dan preferencia a la prueba de trabajo de parto, en EEUU se inclinan por una cesárea electiva. El Colegio Americano de Obstetricia y Ginecología declaro que "una mujer con cesárea anterior debería ser aconsejada de intentar un parto vaginal". En su boletín de práctica señala que la prueba de trabajo de parto post cesárea es una opción segura y apropiada para la mayoría de mujeres que han tenido una cesárea previa. ${ }^{13}$

En esta investigación, se observó que entre los factores maternos enfocados a los antecedentes que presentaron las mujeres con cesárea previa, y estaban relacionados a la decisión de parto vaginal, fueron con mayor frecuencia el período intergenésico y el parto vaginal previo. Este resultado es congruente al encontrado por Arrunátegui G. (17) cuyo estudio se desarrolló en Trujillo, y encontró que el antecedente de parto vaginal es reconocido como factor predictor de parto vaginal exitoso $(\mathrm{OR}=6,41 ; \mathrm{p}<0,01)$ asimismo fue en el período intergenésico óptimo (2-5 años) donde se asoció al parto vaginal exitoso $(\mathrm{OR}=2,69 ; \mathrm{p}<0,05)$. De la misma manera fue para Sepúlveda D., et al, ${ }^{19}$ donde reportó que el antecedente de parto previo la edad materna son factores que se asocian con el éxito del parto en pacientes con cesárea previa. Por su parte Romero J. ${ }^{20}$ el cual desarrollo una investigación en Ecuador sostuvo que el período intergenésico corresponde en un $60 \%$ más de 2 años y en un $40 \%$ entre 1 a 2 años. Por ello el período intergenésico óptimo para prevenir complicaciones maternas y fetales es de 2 a 5 años. Debido a que muchas veces se ha recomendado realizar cesárea en gestantes con período intergenésico menor a 18 
meses, ${ }^{33}$ Otros de los factores maternos enfocados a las características ginecoobstétricas que presentaron las mujeres con cesárea previa, y estaban relacionados a la decisión de parto vaginal, en mayor frecuencia fueron la multigesta, el inicio de parto espontáneo, la multípara y la decisión vía de culminación del médico; resultados que coinciden parcialmente con nuestros resultados donde Arrunátegui G. ${ }^{17}$ estudió los factores predictores para prueba de parto vaginal exitosa en gestantes con cesárea previa, encontrando como factores asociados o predictores de parto vaginal exitosa, la multiparidad.

Desde el inicio de su práctica, la operación cesárea ha ido en un aumento progresivo. Es probable que diversos factores hayan incidido en este incremento, entre los factores estudiados en la presente investigación se encontró que la edad gestacional promedio fue 39,2 $\pm 0,6$ semanas y el promedio ponderado fetal fue de 3,296 \pm 314 gramos; resultados que no alejan de encontrado por De La Vega G, et al ${ }^{41}$ realizaron un reporte de casos en el Hospital Nacional Docente Madre Niño San Bartolomé sobre gestantes a término con el antecedente cada una de haber sido cesareada anterior en 2 ocasiones, a quienes se les ofreció la oportunidad de parto por vía vaginal en la gestación actual, en los tres casos el ponderado fetal fue similar al promedio obtenido en nuestro estudio $3450 \mathrm{~g}, 3350 \mathrm{~g}$, y $3500 \mathrm{~g}$. Por su parte, Zegarra M. ${ }^{42}$ evaluó 512 historias clínicas de gestantes con antecedente de cesárea sometidas a parto vaginal, donde el rango de peso al nacer menor a $4000 \mathrm{gr}$ fue el más frecuente $(93,87 \%)$; solo dos mujeres tuvieron recién nacidos de peso al nacer mayor a 4500 gr. En este estudio se concluyó que los recién nacidos con menos de 4000 gr tienen 6,8 veces más probabilidades de éxito para el parto vaginal. Estos hallazgos coinciden con lo encontrado en la literatura que reporta, que factores como un peso mayor de $4000 \mathrm{gr}$ al nacimiento indicarían un fracaso en el intento de parto vaginal y un aumento en las complicaciones

\section{REFERENCIAS BIBLIOGRÁFICAS}

1. Society of Obstetricians and Gynaecologists of Canada (SOGC). Guidelines Society of Obstetricians and Gynaecologists of Canada (SOGC). Guidelines for vaginal birth after previous cesarean birth: No. 155. J Obstet Gynaecol Can. 2005; 27: 164-74.

2. López M, Sánchez I. Parto después de cesárea anterior: a propósito de un caso. Revista de Enfermería Docente. 2014; 102: 45-51.

3. Organización Mundial de la Salud. Comunidado de prensa: Solo se deben practicar cesáreas que sean necesarias por motivos médicos. Suiza [internet]: Centro de Prensa de la OMS [fecha de acceso 8 de abril de 2016]. 2015.

4. Deneux T, Carmona E, Bouvier C, Bréart G. Postpartum maternal mortality and cesarean delivery. Obstetrics \& Gynecology. 2006; 108 (3): $541-548$.

5. Narro J, Hernández J, Imaz V, Silva J, Olea J, Soto G, Ruiz A. Frecuencia de cesáreas en cuatro hospitales del Distrito federal: 20 años después. Ginecol. Obstet. Méx. 2005; 73: 291-296.

6. Echais J. Cesárea, experiencia en el hospital general de 
Oxapampa. Sociedad Peruana de Obstetricia y Ginecología. Ginecol. Obstet. 2004; 50 (2): 106-10.

7. Thede L. Unnecessary C-sections. Am J Nurs. 2007; 107 (12):1318.

8. American College of Obstetricians and Gynecologists. Natural birth after previous cesarean delivery. ACOG. Practice Bulletin 5o ed. Washington D.C.: 1999.

9. Organización Panamericana de la Salud. La cesárea solo debería realizarse cuando es medicamente necesaria.

Washington D.C. (United States of America): Oficina Regional para las Américas de la Organización Mundial de la Salud. 2015.

10. Medina S, Ortiz A. Incidencia e indicaciones de cesáreas. Revista de Posgrado de la Vla Cátedra de Medicina [Internet]. 2011 Jun; 207:pp.11-

15. Disponible en:

http://med.unne.edu.ar/revista/revista207/3_207.pdf

11. Santaeulalia I. El uso abusivo de cesáreas en América Latina contradice a la OMS. México [internet]: Diario El País [fecha de acceso 8 de abril de 2016]. 2013.

12. Organización Mundial de la Salud. Declaración de la OMS sobre las tasas de cesárea. Ginebra [internet]: Departamento de Salud Reproductiva e Investigación. 2015. .pdf

13. ACOG Practice bulletin no. 115: Vaginal birth after previous cesarean delivery. Obstet Gynecol 2010; 116: 450-463.

14. Grobman W, Lai Y, Landon M, Spong C, Leveno K, Rouse D, et al. Can a prediction model for vaginal birth after cesarean also predict the probability of morbidity related to a trial of labor? Am J Obstet Gynecol 2009; 200: 56- 60.

15. Quispe A, Santibáñez A, Leyton I, Pomasunco D. Cesáreas en siete hospitales públicos de Lima: Análisis de tendencia en el período 2001- 2008. Rev Per Med Exp Salud Pública. 2010; 7 (1): 45-50.

16. Sánchez D. Impacto de la cesárea sobre la salud materna y perinatal; Revisión sistemática [Tesis para optar título de especialista en ginecología y obstetricia]. Colombia: Universidad del Rosario. 2012. Disponible en: http://repository.urosario.edu.co/bitstream/handle/10336/3191/528 60332- 2012. pdf? sequence $=4$

17. Arrunátegui G. Factores predictores para prueba de parto vaginal exitosa en gestantes con cesárea previa en el Hospital de Especialidades Básicas la Noria. [Tesis para optar título de médico cirujano]. Trujillo-Perú: Universidad Privada Antenor Orrego. 2015.

18. Vargas A, Lévano J, Ángeles M. Parto vaginal después de una cesárea, aplicando un puntaje al momento del ingreso a un hospital. Rev Peru Ginecol Obstet. 2013; 59 (4): 261-266.

19. Sepúlveda D, Galván M, Soto G, Méndez D. Factores asociados con éxito en el parto de mujeres con antecedente de cesárea. 
Ginecol Obstet Mex 2015; 83: 743-749.

20. Romero J. Determinación de los factores favorables para un parto vaginal post cesárea en primigestas juveniles de 15 a 20 años en el Hospital Dra. Matilde Hidalgo Procel. De septiembre de 2012 a febrero de 2013. [Tesis para optar título de obstetra]. Guayaquil (Ecuador): Universidad de Guayaquil; 2013.

21. Castillo M, Arenas J. Muñoz P, Santos M, López P, Castaño L. Factores relacionados con la vía de parto tras cesárea anterior. Clínica e Investigación en Ginecología y Obstetricia. 2009; 36 (4):122-126.

22. Macones G, Peipert J. Maternal complications with vaginal birth after cesarean delivery: a multicenter study. Am J Obstet Gynecol. 2005; 193: 1656-1662.

23. Landon M, Hauth J. Maternal and perinatal outcomes associated with a trial of labor after prior cesarean delivery. National Institute of Child Health and Human Development Maternal-Fetal Medicine Units Network. N Engl J Med 2004; 351: 2581-2589.

24. Eden K, McDonagh M, Denman MA, Marshall N, Emeis C, Fu R, Janik $R$, et al. New insights on vaginal birth cesarean. Can it be predicted? Obstet Gynecol. 2010; 116 (4):967-81.

25. Instituto Mexicano de Seguridad Social. Guía de Práctica Clínica: Parto después de una cesárea. México: Consejo de Salubridad General.2013.

26. Society of Obstetricians and Gynaecologists of Canada (SOGC). Guidelines for vaginal birth after previous cesarean birth: No. 155. J Obstet Gynaecol Can. 2005; 27: 164-174.

27. Royal College of Obstetricians and Gynaecologists (RCOG). Birth After Previous Caesarean Birth: Green-Top Guideline No. 45. London: Royal College of Obstetricians and Gynaecologists, 2007.

28. Bujold E, Gauthier R. Risk of uterine rupture associated with an interdelivery interval between 18 and 24 months. Obstet Gynecol. 2010; 115: 1000-6.

29. Guise J, Denman M, Emeis C, Marshall N, Walker M, Fu R, et al. Vaginal birth after cesarean. New insights on maternal and neonatal outcomes. Obstet Gynecol 2010; 115: 1267-78.

30. Colleman T, Randall H, Graves W, Lindsay M. Vaginal birth after cesarean among women with gestacional diabetes. Am J Obstet Gynecol. 2001; 184: 1104-1107.

31. Blackwell S, Hassan S, Wolfe H, Michaelson J, Berry S, Sorokin Y. Vaginal birth after cesarean in the diabetic gravida. J Reprod Med. 2000; 45: 987-990.

32. García C, López M, Monzalbo D. Parto después de cesárea ¿una opción segura? Ginecol Obstet Mex. 2015; 83: 69-87.

33. Huang W, Nakashima D, Rumney $P$, et al. Interdelivery interval and the success of vaginal birth after cesarean delivery. Obstet Gynecol 2002; 99: 41-44.

34. Cahill A, Stamilio D, Odibo A. Is vaginal birth after cesarean (VBAC) or elective repeat cesarean safer in women with a prior 
vaginal delivery? Obstet Gynecol. 2006; 195: 1143-1147.

35. Srinivas $S$, Stamilio D, Stevens E. Predicting failure of a vaginal birth attempt after cesarean delivery. Obstet Gynecol. 2007; 109: 800-805.

36. Mc Donagh M, Osterweil P, Guise J. The benefits and risks of inducing labour in patients with prior caesarean delivery: a systematic review. BJOG 2005; 112: 1007-1015.

37. Crespo R, Alvir A, Lapresta M, Andrés M, Campillos J, Castán S. Impacto de la edad materna avanzada en las complicaciones obstétricas y perinatales de las gestaciones gemelares. Clínica e Investigación en Ginecología y Obstetricia. 2012; 39 (6): 230-235.

38. Olivero S. Sociedad e Economía en San Isidro Colonial. $1^{\underline{a}}$ ed. Madrid: Universidad de Sevilla. 2006. p161.

39. Hacker F, Gambone J, Hobel C. Ginecología y obstetricia de Hacker y Moore. México: Editorial Manual Moderno. 2011.

40. Hernández, R., Fernández, C., y Baptista, P. Metodología de la investigación. (6.a ed.) México: McGraw-Hill. 2014.

41. De la Vega G, Gómez L, Bautista F, Ludmir J. Parto vaginal en cesareada anterior dos veces. Hospital San Bartolomé. Rev. Ginecol. Obstret. 2001; 47 (3): 193-197.

42. Zegarra M. Parto vaginal en cesareada anterior [tesis para optar el título profesional de médico cirujano]. Lima: Universidad Ricardo Palma; 2005. 